\title{
Functionality of nanopatterned polymer surfaces
}

\begin{abstract}
The different methods of the nanopatterning of polymer layers, UV laser ablation, plasma depositing technique, electrochemical deposition and soft lithography are discussed as methods of surface patterning. The different surface functionalities are described, especially the effects of increased surface hydrophobicity/ superhydrophobicity created by coating substrates with low surface energy material coupled with controlling the polymer surface roughness at both micro- and nanoscale, with many of these hydrophobic layers representing bio-inspired surfaces. Cell adhesion onto nanopatterned polymer surfaces, bacteria and biomolecules immobilization, and cancer cell isolation are discussed as switchable functionalities. A new field is self-assembled monolayers formed from polymers, which can modulate a surface functionality from hydrophilicity to hydrophobicity, forming highly ordered molecular structures to bind different biomolecules and to create stimuli-responsive polymer systems. These polymers have the potential to tune surface wettability to a desired level with a controlled surface structure and smoothness. Examples are described of rotaxenes which are polymeric self-assembled monolayers which can form molecular devices/nanodevices.
\end{abstract}

Keywords: nanopatterning, polymer, surface, functionality

\author{
Volume I Issue 4 - 2017
}

\author{
Orinak A,' Orinakova R,' Macko J, ${ }^{1,2}$ Petrus \\ O,' Levoca S' \\ 'Department of Physical Chemistry, University of P J Safarik in \\ Kosice, Slovakia \\ ${ }^{2}$ Department of Physical and Theoretical Chemistry, Komensky \\ University Bratislava, Slovakia
}

Correspondence: Orinak A, University of P J Safarik in Kosice, Srobarova 2, Kosice, Slovakia, Tel 004210552342321,

Email andrej.orinak@upjs.sk

Received: April 17, 2017| Published: August 28, 2017
Abbreviations: DBS, dodecylbenzene sulfonate; PDMS, polydimethylsiloxanes; PEG, polyethyleneglycol; PLA, polylactic acid; PLGA, poly (lactic-co-glycoid acid); PNIPAAm, poly (N-isopropy-lacrylamide); PPy, poly pyrrole; PS, poly styrene; PSS, polysodium styrene sulfonate; QAS, quaternary ammonium salt; SAM, self-assembled monolayers; PDMS, polydimethylsiloxane

\section{Introduction}

The aim of this mini review was zoom in the perspective and feasible methods to produce nanopatterned polymers surfaces and to collect their some properties. This has opened new possibilities for making affordable polymer products with functional nanopatterned surfaces.

While a variety of approaches to create hydrophobic/ superhydrophobic surfaces have been developed, ${ }^{1-5}$ there are still restrictions on their widespread use due to the cost, number of processing steps, limits on the manufacturable area, durability, instrumentation required, etc. This has led to the development of inexpensive and reliable techniques for the commercial production of hydrophobic layers. Electrochemical methods have been used to prepare a $\mathrm{Ni}$ based nanostructured surface coupled with a polydimethylsiloxane (PDMS) monolayer using spin - coating. ${ }^{6}$ The surface hydrophobicity increased by about $30 \%$ in comparison to the $\mathrm{Ni}$ surface only and could be modified to enhance the adhesion of ordered biomolecules or the self-assembly of monolayers. Alternative simple methods included the fabrication of a shark-skin-like patterned PDMS modified with carbon nanotubes to form a polymeric superhydrophobic film. ${ }^{7}$ A high viscosity paste (comprised of $10 \mathrm{wt} \%$ multi walled carbon nanotubes dispersed into PDMS) was placed between two rollers, and the paste was transferred as a smooth film (with less than $300 \mathrm{~nm}$ roughness) onto the roll with the high rotational speed. ${ }^{7}$ A spin-coating method has been used to form a biomimetic interface from melanin whose electric signal transduction can be modified. ${ }^{8}$ High density plasma processing was employed to create a nanotextured superhydrophobic transparent poly (methyl methacrylate) surface. ${ }^{9}$ By appropriate tuning of the plasma conditions, either random or ordered hierarchical structures of high aspect ratio and surface area could be reproducibly created. Such plasma treated polymeric surfaces have been evaluated as substrates for efficiently controlling the wettability, biomolecules immobilization, and cell adhesion of the surface, paving the way to a wide spectrum of applications. A three-dimensional plasma micro-nanotextured cyclo-olefin-polymer surfaces has been used for biomolecules immobilization because of its environmentally stable superhydrophobic and superoleophobic properties..$^{10}$ Plasmas could also be used to deposit fluorocarbons on a cyclo-olefin polymer to enhance hydrophobicity ${ }^{11}$ with good adhesion and effective surface protection reducing plastic deformation. A micro reactive ion-etching method has been used to prepare nanotextured films from PDMS for enhanced cancer cell isolation. ${ }^{12}$

Slippery liquid-infused porous surfaces show great promise for preventing biofilm formation owing to their low surface energy in combination with their self-cleaning properties. ${ }^{13} \mathrm{Li}$ and co-workers demonstrated a novel hydrophobic liquid-infused porous poly (butyl methacrylate-co-ethylene dimethacrylate) surface with bacteriaresistance in BM2 mineral, which had medium and long-term stability in aqueous environments with Pseudomonas aeruginosa. ${ }^{13}$

Highly enhanced solid-state thermochromism was observed in regio-regular poly (3-hexylthiophene) when deposited on a superhydrophobic polymer- $\mathrm{SiO}_{2}$ nanocomposite coating without reducing the superhydrophobicity. ${ }^{14}$ Peng et al. ${ }^{15}$ have quantified the volume and surface hydrophilicity of a range of waterswollen dense polymer brushes as a function of temperature. They used thermo responsive poly ( $\mathrm{N}$-isopropylacrylamide) and poly (di(methoxyethoxy) ethyl methacrylate), strongly hydrophilic poly (N, N-dimethylacrylamide) and poly (oligo (ethylene glycol) methacrylate), and weakly hydrophilic poly (2-hydroxyethyl 
methacrylate) brushes synthesized by surface-initiated atomtransfer radical polymerization. There were two types of behavior: strongly swollen brushes exhibited a progressive decrease in volume hydrophilicity with temperature, while surface hydrophilicity changed moderately; weakly swollen brushes had a close-to-constant volume hydrophilicity, while surface hydrophilicity decreased with temperature. ${ }^{16}$ Altering the surface wettability by external stimulation has received great attention recently. Wagner et al. ${ }^{17}$ have summarized many recent achievements in the field of reversibly switchable polymer films with a focus on changes in wettability. Particularly interesting were light/photo-responsive polymer coatings, which could be used for versatile applications, such as in the biomedical industry or in light emitting diodes, etc. The hydrophilicity of Polylactic acid (PLA) electrospun fibers were modified through the incorporation of polyethylene glycol (PEG) homopolymer and PLA-b-PEG co-polymers of different block lengths by Hendrick. ${ }^{18}$ The lengths of the blocks were found to influence the maximum amount of PEG that could be incorporated, as well as spin-ability and morphology. Water wettability improved significantly with the addition of PEG to the electrospinning dopes, with PLA-b-PEG copolymers providing a greater increase than the PEG homopolymer. PDMS films with 2D periodic inverted moth-eye nanopatterns on upper surface were implemented as antireflection layers on a glass substrate for efficient light capture in encapsulated organic solar cells. Inverted moth-eye PDMS films laminated on glass substrates exhibit a hydrophobic surface with a water contact angle of $\approx 120^{\circ} \mathrm{C}$ and solar weighted transmittance of $\approx 94.2 \%{ }^{19}$ Very similar antireflective hydrophobic polystyrene surfaces have been prepared by Kuo et al. ${ }^{20}$ using the methods of Langmuir-Blodgett deposition and nanoimprint lithography. Ito et al. ${ }^{21}$ described the superhydrophobicity of the transparent typical polymers, such as polycarbonate and polystyrene patterned with nanorod and hierarchical structure, which combined nanorods with micro convex structures.

Chu et al. ${ }^{22}$ examined systematic patterned hierarchical structures with dual scale roughness that has been formed using simple and large-scale micro-manufacturing methods. The authors evaluated the apparent contact angles to determine the effect of geometric surface parameters on the hydrophobic properties and found that contact angles of more than $130^{\circ} \mathrm{C}$ could be obtained without additional coatings. Yu et al. ${ }^{23}$ prepared surfaces with switchable functionality based on nanopatterned, thermo responsive poly ( $\mathrm{N}$-isopropylacrylamide) (PNIPAAm) brushes. They were fabricated using interferometric lithography combined with surface-initiated polymerization. The temperature-triggered conformation changes reversibly modulated the spatial orientation of the polymer chains which leads to the hiding or exposure of molecules that were immobilized in the intervals between the nanopatterned brushes. A biocidal quaternary ammonium salt (QAS) was used to demonstrate the utility of the nanopatterned PNIPAAm brushes to control biointerfacial interactions with bacteria. QAS was integrated into polymer-free regions of the substrate. Above the lower critical solution temperature of PNIPAAm, the polymer chains exposure quarternary ammonium salts that killed attached bacteria. Below the critical temperature, the PNIPAAm chains promoted the release of dead bacteria. Golabi et al. ${ }^{24}$ studied the physical-chemical properties of polypyrrole (PPy) electrochemically prepared with different dopants $\left(\mathrm{Cl}, \mathrm{ClO}_{4}\right.$, p-toluene-sulfonate (ToS), dodecylbenzene sulfonate (DBS), and poly sodium styrene sulfonate (PSS)) under two applied potentials. E. coli showed greater adhesion tendency to the hydrophobic, rough surface of PPy-DBS, and less adhesion to the smooth and hydrophilic surface of PPy-PSS. Blends of hydrophobic polystyrene (PS) and hydrophilic acrylonitrile butadiene styrene polymers with different weight ratios, modified by avidin, to modify their surface chemistry and topography were prepared by Juvonen and co-workers ${ }^{25}$ to study the adhesion of Staphylococcus aureus. When the surface coverage of PS component was $\sim 85 \%$ the bacterial adhesion was increased and was suitable for using in sensing applications. However, when the surface coverage of PS component was $\sim 55 \%$ the adhesion of bacteria was inhibited. This result may open a new way for the development of surfaces with potential antimicrobial properties.

Treatment of polymer surface can be a simple and quick method to produce nanostructured polymer surfaces with anti-bacterial properties. Karahaliloglu et al. ${ }^{26}$ used alkaline surface treatment to create random nano objects on poly (lactic-co-glycoid acid) (PLGA) membranes, where the nanomorphology of PLGA was affected by the $\mathrm{NaOH}$ concentration and exposure time. The most significant results showed a decrease in $S$. aureus growth, without antibiotics, of up to $\sim 40 \%$ after 2 days, following alkaline treatment PLGS $(0.5 \mathrm{M}$ $\mathrm{NaOH} ; 10$ min) compared to untreated controls. The nanostructured PLGA membranes have a much higher wettability with altered surface functional groups. The newly formed membranes promoted keratinocyte adhesion and proliferation, while limiting fibroblast functions and bacterial growth. Another group ${ }^{27}$ published bacterial studies showed that nano-PDMS significantly inhibited the growth of $S$. aureus and E. coli after 48 h without using antibiotics, while remaining non-toxic to fibroblasts and endothelial cells.

The combination of colloidal lithography with reactive ion etching enabled Liu et al. ${ }^{28}$ to prepare PET nanocone arrays with underwater superoleophobicity and anti-bioadhesion properties. The nanocone array with aspect ratio value of 6 had superoleophobicity with oil contact angle of $171.8{ }^{\circ} \mathrm{C}$ and had anti-adhesion behavior, when it was used for cell culture cultivation. Sánchez-Salcedo et al. ${ }^{29}$ tested zwiterionic polymers on ceramic powder and metallic powders for application in bone tissue engineering. The polymers on metal nanostructured surfaces showed excellent inhibition (99\%) of the adhesion of $E$. coli compared to unmodified substrates.

Improving the biocompatibility of biomaterial surfaces by grafting on polymers brings promising effects. Graft polymerization does produce sufficiently grafted polymers, hence leads to nonspecific protein adsorption. ${ }^{30,31}$ Covalent coupling produced a stable attachment of polymers for long term applications but can be circumvented by modifying surfaces with self-assembled monolayers (SAM) of organic molecules, such as thiols or silanes, which provided covalent interfaces between substrate and polymer(s). This method offered a molecularlevel control of material structure, composition, morphology and dimensions, thus enabling the construction of an extensive variety of 1-, 2- and 3D nanostructures. ${ }^{32-35}$ Efforts have been carried out to elaborate processes able to fabricate organized materials with nanometer resolution, and these have found application in protective coatings, ${ }^{36}$ molecular electronic devices, ${ }^{37,38}$ sensors, ${ }^{39,40}$ robust textiles ${ }^{41}$ and biofunctional materials. ${ }^{42}$ One example is the rotaxanes, containing a pyridine function in the macrocyclic component, which can be grafted onto carboxylic acid-terminated SAMs, giving rise to monolayers or sub-monolayers of rotaxanes. They are attached to the carboxylic acid-terminated surface by hydrogen-bonding between the pyridine group of the macrocycle and the carboxylic acid group of the $\mathrm{SAM}^{43}$ Several stimuli, such as $\mathrm{pH}$, temperature and even light, 
can be used to initiate a structural reconfiguration driven by weak hydrogen-bonds. ${ }^{44}$

\section{Discussion}

The nanopatterning of polymeric surfaces has been shown to be a method for the fabrication of highly or randomly ordered structures at the nano level that have shown good functionality in many fields of science. Methods for the cheaper preparation of such functionalized layers for wide range of purposes are still being developed, including surface hydrophobicity modulation for anti-fouling and cell adhesion, as well coatings with bio-mimetic properties. Despite recent progress it is important to improve the surface ant adhesion properties of medical devices to prevent bacterial colonization.

A unique nanohybrid surface with a marked increase in surface hydrophobicity has been observed on the differently polar polymer layers, which have been spin-coated directly on a previously prepared nanostructured nickel surface to form nanohybrids. Nanostructured nickel layers have also been prepared by electrochemical deposition to form a random polyhedral crystalline nanostructure (Figure 1). The hydrophobicity of the surface can be modulated with the roughness of the surface and the polarity of the covering polymer surface. The method is very economical and does not require expensive equipment. If PDMS was used as the coating, the nanohybrid surface blocked the adhesion and proliferation of living cells. Similar smart polymer surfaces were also prepared by more expensive methods using laser electron beam lithography. ${ }^{45}$ However, the mechanical stability of surfaces with PDMS is still problematic. Unpublished results with polyethylene monolayer showed better resistance to abrasion. Such modified surface resulted in a self-cleaning property.

\section{A}

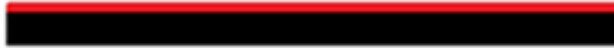

Stainless steel Polymer layer

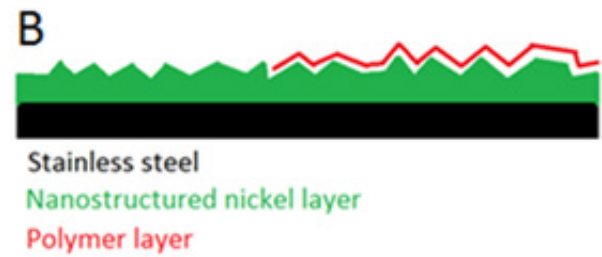

Figure I Cross-sectional picture of newly prepared nanostructured nickelpolymer nanohybrids.

A: Single polymer on the stainless steel layer.

B: Polymer layer on a surface of nanostructured nickel template to form nanohybrid.

The direct patterning of polymer surface with highly ordered and oriented nanoparticles (nanorods, nano pillars and others) appears to be not commercially viable. Mentioned are also studies that have been carried out to produce artificial biomimetic roughness-induced hydrophobic surfaces. ${ }^{46}$ To explain effect of nano- or micro- patterning or both on surface ${ }^{47,48}$ hydrophobicity is also often examined. Selfassembly of random copolymers has recently attracted considerable attention, to prepare nanostructures with different morphologies and nanomaterials that are responsive to single or multiple stimuli. Era of SAM brings exploitation of the molecular devices and nanodevices ${ }^{49}$ shown in Figure 2.

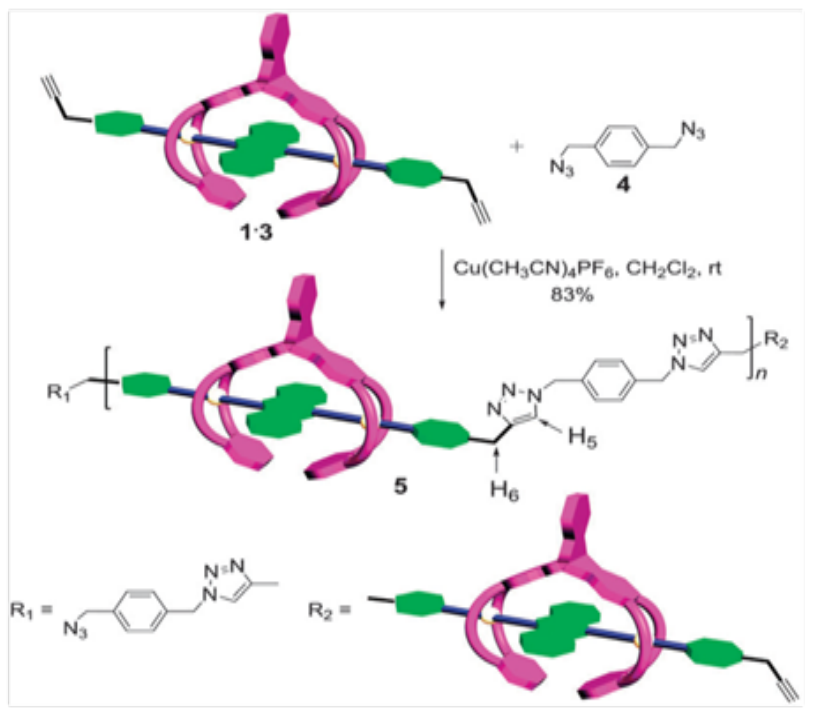

Figure 2 Scheme of synthesis of polymeric polyrotaxanes. Reproduced from ${ }^{50}$ with permission from The Royal society of chemistry.

\section{Conclusion}

The ability to pattern functional polymers at different lengths is important in many research fields, including cell biology, tissue engineering and medicinal science and the development of optics and electronics. The interest and capabilities of polymer patterning have originated from the abundance of functionalities of polymers and a wide range of applications of the patterns. This review considers recent advances in the functionality of the patterning of polymers using different methods and self-assembly of block copolymers. The main functions of such prepared surfaces included surface hydrophobicity, adhesion strength and SAM application leading to molecular devices with specific functions. Finally, challenges and future directions are discussed from the point of view of both applicability and strategies for the surface patterning of polymers.

\section{Acknowledgements}

The authors are grateful for the financial support from MS SR VEGA and 1/0074/17 and APVV-16-0029. Authors wish to thank Roger M. Smith for manuscript editing.

\section{Conflicts of interest}

The author declares no conflict of the conflicts.

\section{References}

1. Neinhuis C, Barthlott W. Characterization and distribution of water-repellent, self-cleaning plant surfaces. Ann Bot. 1977;79:667-677.

2. Erbil HY, Demirel AL, Avci Y, et al. Transformation of a simple plastic into a superhydrophobic surface. Science. 2003;299(5611):1377-1380.

3. Nakajima A, Fujishima A, Hashimoto K, et al. Preparation of transparent superhydrophobic boehmite and silica films by sublimation of aluminum acetylacetonate. Adv Mater. 1999;11(16):1365-1368. 
4. Cao L, Jones AH, Sikka VK, et al. Anti-icing superhydrophobic coatings. Langmuir. 2009;25(21):12444-12448.

5. Sun TL, Tan H, Han D, et al. No platelet can adhere-largely improved blood compatibility on nanostructured superhydrophobic surfaces. Small. 2005;1(10):959-963.

6. Macko J, Oriňak A, Oriňaková R, et al. New nanostructured nickel-polymer nanohybrids with improved surface hydrophobicity and effect on the living cells adhesion. Appl Surf Sci. 2015;355:553-561.

7. Park S H, Lee S, Moreira D, et al. Bioinspired superhydrophobic surfaces, fabricated through simple and scalable roll-to-roll processing. Sci Rep. 2015;5:15430

8. Ambrico M, Ambrico PF, Cardone A, et al. Melanin-like polymer layered on a nanotextured silicon surface for a hybrid biomimetic interface. J Mater Chem C. 2014;3:573-582.

9. Ellinas K, Tsougeni K, Petrou PS, et al. Three-dimensional plasma micro-nanotextured cyclo-olefin-polymer surfaces for biomolecule immobilization and environmentally stable superhydrophobic and superoleophobic behavior. Chemical Engineering Journal. 2016;300:394-403.

10. Vourdas N, Tserepi A, Gogolides E. Nanotextured super-hydrophobic transparent poly (methyl methacrylate) surfaces using high-density plasma processing. Nanotech. 2007;18(12):125304.

11. Dragatogisnnis A, Koumoulos E, Ellinas K, et al. Nanoscale mechanica and tribological properties of plasma nanotextured COP surfaces with hydrophobic coatings. Plasma Process Polym. 2015;12(11):1271-1283.

12. Islam M, Sajid A, Mahmod MAI, et al. Nanotextured polymer substrates show enhanced cancer cell isolation and cell culture. Nanotechnology. 2015;26(22):225101.

13. Li J, Kleintschek T, Rieder A, et al. Hydrophobic liquid-infused porous polymer surfaces for antibacterial applications. ACS Appl Mater Interfaces. 2013;5(14):6704-6711.

14. Cataldi P, Bayer IS, Cingolani R, et al. A thermochromic superhydrophobic surface. Sci Rep. 2016;6:27984.

15. Peng CW, Chang KC, Weng CJ, et al. UV-curable nanocasting technique to prepare bio-mimetic super-hydrophobic non-fluorinated polymeric surfaces for advanced anticorrosive coatings. Polym Chem. 2013;4:926930.

16. Zhuang P, Dirani A, Glinel K, et al. Temperature dependence of the surface and volume hydrophilicity of hydrophilic polymer brushes. Langmuir. 2016;32(14):3433-3444.

17. Wagner N, Theato P. Light-induced wettability changes on polymer surfaces. Polym. 2014;55(16):3436-3453.

18. Hendrick E, Frey M. Increasing surface hydrophilicity in poly (lactic acid) electrospun fibers by addition of PLA-b-PEG Co-Polymers. J Eng Fabr Fibers. 2014;9(2):153-164.

19. Leem JW, Kim S, Lee SH, et al. Efficiency enhancement of organic solar cells using hydrophobic antireflective inverted moth-eye nanopatterned PDMS films. Adv Energy Mater. 2014;4(8):1-7.

20. Kuo WK, Hsu JJ, Nien CK, et al. Moth-eye-inspired biophotonic surfaces with antireflective and hydrophobic characteristics. ACS Appl Mater Interfaces. 2016;8(46):32021-32030.

21. Ito H, Chu D, Takayama T, et al. Micro- and nano-hierarchical structures on polymer surfaces for application to superhydrophobic properties. Int Work Microfactories. 2014;(5-8):30-33.

22. Chu D, Nemoto A, Ito H. Biomimetic superhydrophobic polymer surfaces by replication of hierarchical structures fabricated using precision tooling machine and anodized aluminum oxidation. Microsyst Technol. 2014;21(1):123-130.
23. Yu Q, Cho J, Shivapooja P, et al. Nanopatterned smart polymer surfaces for controlled attachment, killing, and release of bacteria. ACS Appl Mater Interfaces. 2013;5(16):9295-9304.

24. Golabi M, Turner A, Jager E. Tuning the surface properties of polypyrrole films for modulating bacterial adhesion. Macromol Chem Phys. 2016;217(10):1128-1135.

25. Juvonen H, Oja T, Maattanen A, et al. Protein and bacterial interactions with nanostructured polymer coatings. Colloids Surfaces B Biointerfaces. 2015;136:527-535.

26. Karahaliloglu Z, Ercan B, Chung S, et al. Nanostructured anti-bacterial poly-lactic-co-glycolic acid films for skin tissue engineering applications. J Biomed Mater Res Part A. 2014;102(12):4598-4608.

27. Liu L, Ercan B, Sun L, et al. Understanding the Role of Polymer Surface Nanoscale Topography on Inhibiting Bacteria Adhesion and Growth. ACS Biomater Sci Eng. 2016;2(1):122-130.

28. Liu W, Liu X, Fangteng J, et al. Bioinspired polyethylene terephthalate nanocone arrays with underwater superoleophobicity and anti-bioadhesion properties. Nanoscale. 2014;6(22):13845-13853.

29. Sánchez Salcedo S, Colilla M, Izquierdo Barba I, et al. Preventing bacterial adhesion on scaffolds for bone tissue engineering. Int J Bioprinting. 2016;2(1):20-34.

30. Jeon SI, Andrade JD. Protein-surface interactions in the presence of polyethylene oxide: II. Effect of protein size. J Colloid Interface Sci. 1991;142:159-166.

31. Jeon SI, Lee JH, Andrade JD, et al. Protein-surface interactions in the presence of polyethylene oxide: I. Simplified theory. J Colloid Interface Sci. 1991;142:149-158.

32. Palmer LC, Stupp SI. Molecular self-assembly into one-dimensional nanostructures. Acc Chem Res. 2008;41(12):1674-1684.

33. Capito RM, Azevedo HS, Velichko YS, et al. Self-assembly of large and small molecules into hierarchically ordered sacs and membranes. Science. 2008;319(5871):1812-1816.

34. Zubarev ER, Pralle MU, Sone ED, et al. Self-assembly of dendron rodcoil molecules into nanoribbons. J Am Chem Soc. 2001;123(17):41054106.

35. Jiyun C H. Guided molecular self-assembly: A review of recent efforts. Smart Mater Struct. 2003;12(2):264-275.

36. Amigoni S, Taffin de Givenchy E, Dufay M, et al. Covalent layer-by-layer assembled superhydrophobic organic-inorganic hybrid films. Langmuir. 2009;25(18):11073-11077.

37. Minari T, Liu C, Kano M, et al. Controlled self-assembly of organic semiconductors for solution-based fabrication of organic field-effect transistors. Adv Mater. 2012;24(2):299-306.

38. Halik M, Hirsch A. The potential of molecular self-assembled monolayers in organic electronic devices. Adv Mater. 2011;23(22-23):26892695.

39. Xie G, Sun P, Yan X, et al. Fabrication of methane gas sensor by layer-by-layer self-assembly of polyaniline/PdO ultrathin films on quartz crystal microbalance. Sens Actuators B. 2010;145:373-377.

40. Bussetti G, Violante A, Yivlialin R, et al. Site-sensitive gas sensing and analyte discrimination in Langmuir-Blodgett porphyrin films. $J$ Phys Chem C. 2011;115(16):8189-8194.

41. Li YC, Mannen S, Morgan AB, et al. Intumescent all-polymer multilayer nanocoating capable of extinguishing flame on fabric. Adv Mater. 2011;23(34):3926-3931. 
42. Zhang S. Fabrication of novel biomaterials through molecular self-assembly. Nat Biotech. 2003;21(10):1171-1178.

43. Dhotel A, Chen Z, Delbreilh L, et al. Molecular motion in functional self-assembled nanostructures. Int J Mol Sci. 2013;14(2):2303-2333.

44. Tokarev I, Minko S. Stimuli-responsive hydrogel thin films. Soft Matter. 2009;5(3):511-524.

45. Brétagnol F, Ceriotti L, Valsesia A, et al. Fabrication of functional nano-patterned surfaces by a combination of plasma processes and electron beam lithography. Nanotech. 2007;18(13):135303-13510.

46. Jung Y Ch, Bhushan B. Contact angle, adhesion and friction properties of micro-and nanopatterned polymers for superhydrophobicity. Nanotech. 2006;17(19):4970-4985.
47. Lassen B, Johansson A, Elwing H. some model surfaces made by RF plasma aimed for the study of biocompatibility. Clin Mater. 1992;11:99103.

48. Kiss E, Golander CG. Chemical derivatization of muscovite mice surface. Colloid Surf. 1990;49:335-342.

49. Zeng F, Meng Y, Han Y, et al. Formation of a "pseudosuitane"- type complex between a triptycene-derived bis(crown ether) host and 1,1-(anthracene-9,10-diyl)bis(N-benzylmethanaminium): a new method for the synthesis of linear polyrotaxanes. Chem Commun. 2014;50(57):76117613. 